\title{
On the Sensor Choice and Data Analysis for Classification of Elementary Upper Limb Movements
}

\author{
Dwaipayan Biswas, Andy Cranny, Ahmed Rahim, Nayaab Gupta, Nick Harris, Koushik Maharatna, \\ IEEE Member, and Steffen Ortmann
}

\begin{abstract}
In this paper we present a systematic exploration for determining the appropriate type of inertial sensor and the associated data processing techniques for classifying four fundamental movements of the upper limb. Our motivation was to explore classification techniques that are of low computational complexity enabling low power processing on body-worn sensor nodes for unhindered operation over a prolonged time. Kinematic data was collected from 18 healthy subjects, repeating 20 trials of each movement, using tri-axial accelerometers and tri-axial rate gyroscopes located near the wrist. Ten time-domain features extracted from data from individual sensor streams, their modulus and specific fused signals, were used to train classifiers based on three learning algorithms: LDA, QDA and SVM. Each classifier was evaluated using a leave-one-subject-out strategy. Our results show that we can correctly identify the four arm movements, with sensitivities in the range of $83-96 \%$, using data from just a tri-axial gyroscope located near the wrist, and requiring only 12 features in combination with the lower complexity LDA learning algorithm.
\end{abstract}

\section{INTRODUCTION}

A Wireless Body Area Network (WBAN) integrating heterogeneous low-cost physiological data measurement sensors in a body-worn system paves a new way for remote healthcare monitoring [1]-[2]. One of the potential application areas of such a system is human activity recognition which has relevance in Stroke rehabilitation within the home environment. The primary requirement of such a system is to ensure its long-term operation for subject monitoring. It has been shown that in wearable systems, the data analysis primarily needs to be carried out at the sensor node yielding energy efficient solutions as compared to conventional remote monitoring approaches based on continuous transmission of data to a central server. In general, these systems are battery powered and are therefore resource constrained, so this efficiency can only be achieved by selecting low-complexity data processing algorithms, since the computational complexity is proportional to the energy consumption [3]. Thus, with respect to remote healthcare, a credible monitoring system should have low

This work was supported by the European Union under the Seventh Framework Programme, grant agreement \#288692, StrokeBack.

D. Biswas, A. Cranny, N. Gupta, N. Harris and K. Maharatna are with the School of Electronics and Computer Science, University of Southampton, Hampshire, SO17 1BJ, UK (e-mail: \{db9g10, awc, ng2g10, nrh, km3\}@ecs.soton.ac.uk).

A. Rahim was studying at University of Southampton and is currently working with Imagination Technologies (e-mail: een5afr@gmail.com).

S. Ortmann is with IHP, Leibniz-Institute for Innovative Microelectronics, Frankfurt (Oder), Germany (e-mail: ortmann@ihpmicroelectronics.com). complex data processing to extend battery life. This presents a challenge when detecting fine-grain activities e.g., upper limb movement, as opposed to detection of gross activities such as cycling or running since the former usually requires higher complexity processing [2].

Typically low-cost inertial sensors (accelerometers and gyroscopes) and magnetometers are used for activity recognition [2]. Radio-frequency identification (RFID) has been used to monitor the movement of objects with which a subject interacts within the home environment [4]. Vision based systems have also been used in activity recognition, but they tend to involve high complexity image processing algorithms, are usually restricted to indoor activities, and require un-hindered surveillance of the subject [5].

In principle there are three steps for activity recognition using inertial sensors: 1) data capture by appropriate sensor; 2) segmentation of the captured data to identify the beginning and end of an activity and 3) recognition of the activity using appropriate classification techniques. Although the final two steps are in practise interrelated, individually they pose significant research challenges owing to the possible qualitative non-uniqueness of an activity pattern exhibited by an individual subject. Therefore these are treated as two individual research problems: event detection and activity recognition. We concentrate only on the second research problem, activity recognition, on the assumption that the start and stop time of the activity is known.

In this paper we report on a systematic exploration aimed at accurately recognising four fundamental (fine-grain) movements of the upper limb that are associated with basic activities of daily living. We are particularly interested in developing a low-complexity, robust training model that accounts for significant inter-subject variability and we evaluate the model with a 'leave-one-subject-out' validation process, with the aim of determining: 1) appropriate type of sensor to be used and 2) associated data processing and classification methodology that may allow long-term monitoring using a body-worn system. We used data from a tri-axial accelerometer and a tri-axial rate gyroscope attached to the wrist, extracting 10 features from each individual sensor-axes and combinations of these signals as inputs for a number of different classification algorithms. Our results show that the tri-axial rate gyroscope can independently recognise all four arm movements considered here with sensitivities for detection in the range of $83-96 \%$ using 12 (out of a possible 30) time domain features extracted from the three sensor axes $(3 \times 10$ features $)$ using the Linear Discriminant Analysis (LDA) classifier. 


\section{EXPERIMENTAL PROTOCOL}

We selected four elementary types of arm movement typically used during daily activities, these were:

- Movement $A$ - Reach and retrieve an object monitoring extension and flexion of the forearm.

- Movement B - Lift cup to mouth and return to table focusing on rotation of the forearm about the elbow.

- Movement $C$ - Reach out for an object sideways by swinging arm $90^{\circ}$ in horizontal plane and return.

- Movement $D$ - Rotate wrist with arm fully extended through $90^{\circ}$ and return.

Experiments were performed with 18 healthy subjects (age range 24 to 50, male and female, both left and right arm dominant) each subject performing 20 trials of each arm movement separated into four groups of five repetitions with each group of trials being separated by approximately three minutes, to avoid the effects of unconscious self-learning of the activities. The subjects performed the movements in a seated position in the laboratory, with no restrictions on physical factors like seating position, table arrangement and the time required to complete the movements, hence ensuring a wider range of variability in the data paving the way for a robust arm movement classification system.

The commercially available Shimmer 9DoF wireless kinematic sensor module comprising mutually orthogonal triaxial accelerometers, rate gyroscopes and magnetometers, was used as the sensing platform [6]. For our experiments we exclude the magnetometer since it can be affected by the presence of ferromagnetic materials which are expected to be present in the natural environment [7]. The dorsal side of the forearm proximal to the wrist on the dominant arm was chosen as the sensing position since it was likely to produce significant sensor responses to the arm movements being investigated. The XY plane of the sensor module was in contact with the dorsal side of the forearm, AccX (GyroY) points toward the hand and AccZ (GyroZ) points away from the dorsal aspect. Sensor data is collected at a rate of $50 \mathrm{~Hz}$, deemed sufficient for assessing habitual limb movement which is on the higher side compared to assessing holistic activity as in [8]-[9]. The accelerometer and gyroscope ranges are selected at $\pm 1.5 \mathrm{~g}$ and $\pm 500 \% \mathrm{sec}$ respectively.

\section{DATA PROCESSING}

The key steps involved in our data processing are illustrated in Fig. 1 and described in the following sections.

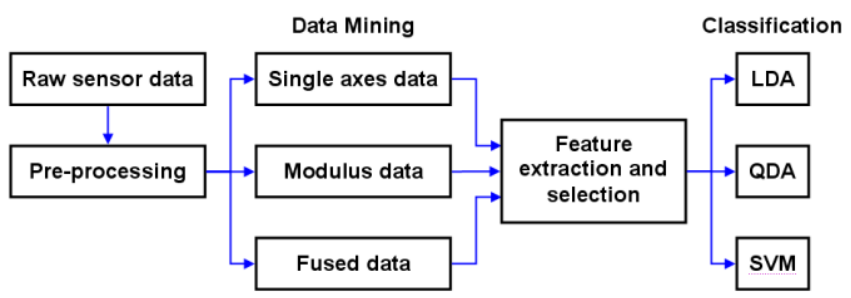

Fig. 1. Methodology used to evaluate data types and learning algorithms.
Acquisition \& Pre-processing - Individual sensors transmit kinematic data along with a time stamp to a host computer using the Bluetooth wireless standard and each activity performed by a subject is marked to record the start and end of the movement during the trial. The raw sensor data is band-pass filtered with a 3rd order Butterworth filter having cut-off frequencies of $12 \mathrm{~Hz}$ and $0.1 \mathrm{~Hz}$ to attenuate the high frequency noise components and low frequency artefacts introduced in the data due to physical effects such as drift [8].

Data Mining - The sensor module transmits data in realtime from a total of 6 individual sensors $(3 \mathrm{x}$ accelerometers and $3 \mathrm{x}$ gyroscopes). We also generate data signals that represent the modulus of the total acceleration $\left(\mathrm{M}_{\mathrm{a}}\right)$ or total rate of rotation $\left(\mathrm{M}_{\mathrm{g}}\right)$ experienced by the forearm as given by Eqn. (1) resulting in two new signals.

$$
\begin{aligned}
& M_{a}=\sqrt{A c c X^{2}+A c c Y^{2}+A_{c c Z}^{2}} \\
& M_{g}=\sqrt{\text { GyroX }^{2}+\text { GyroY }^{2}+\text { GyroZ }^{2}}
\end{aligned}
$$

We further create fused signals, combining data from specific accelerometer-gyroscope axes (see Table I), that are expected to be the most active based on a consideration of the expected trajectory of the subject's arm in relation to the sensor position at the wrist and the orientation of the sensor axes when performing the required movements.

Feature Extraction - Each sensor exhibits signal patterns that are distinctive for each of the arm movements, characterised by a set of features which are extracted from the signals [8]. We consider 10 time-domain features, chosen from amongst those most commonly used in human activity recognition: 1) standard deviation, 2) root mean square (rms), 3) information entropy [10], 4) jerk metric [11], 5) peak number, 6) maximum peak amplitude, 7) absolute difference - absolute difference between the maximum and the minimum value of a signal, 8) index of dispersion, 9) kurtosis, 10) skewness. Although the last two features are usually associated with defining the shape of a probability distribution, they can still be used as classifying features if they routinely return values that distinguish one pattern of data from another. All the 10 features are extracted from each of the six individual sensor data streams (AccX, AccY, AccZ, GyroX, GyroY and GyroZ) and from the two modulus signals $\left(\mathrm{M}_{\mathrm{a}}\right.$ and $\left.\mathrm{M}_{\mathrm{g}}\right)$, as well as from the three fused data signals described in Table I.

TABLE I.

DEFINITION OF FUSED SIGNALS FOR EACH ARM MOVEMENT.

\begin{tabular}{|c|c|}
\hline Movement & Signal combination \\
\hline $\mathrm{A}$ & $\mathrm{Acc} \mathrm{X} \times \mathrm{GyroY}$ \\
\hline $\mathrm{B}$ and $\mathrm{C}$ & $\mathrm{Acc} \mathrm{Y} \times \mathrm{GyroZ}$ \\
\hline $\mathrm{D}$ & $\mathrm{AccZ} \times \mathrm{GyroY}$ \\
\hline
\end{tabular}

Feature Selection - We normalize the extracted features and then follow the Wrapper approach using the lowcomplexity sequential forward selection $(s f s)$ technique [12] as opposed to other multi-class feature ranking/selection algorithms commonly used in the field of human activity recognition, like the ReliefF algorithm [11] and the Clamping technique [9], both of which are computationally 
intensive by comparison. The methodology used selects various feature vector combinations to test for the minimal classification error probability, thereby selecting the best features for a given classification algorithm. Features are selected from: a) data from individual $\mathrm{X}, \mathrm{Y}$ and $\mathrm{Z}$ axes $(3 \times 10$ features) for the accelerometer and gyroscope, b) modulus signals $(1 \times 10$ features $)$ from both the accelerometer and gyroscope, and c) fused signals $(3 \times 10$ features $)$. Hence the number of optimal features selected depends on the classification algorithm employed and the data source applied upon, which has been thoroughly explored here.

Classification - In terms of activity classification, a wide variety of learning techniques have been used e.g. Artificial Neural Networks (ANN) [1], Decision Trees (DT) [1], [8], Support Vector Machines (SVM) [8], Multi-Layer Perceptron (MLP) [9] or a combination of these techniques [5]. From the perspective of low/moderate computational complexity, we restrict our study to three different classifiers - LDA, Quadratic Discriminant Analysis (QDA) and Support Vector Machine (SVM). We used the toolbox LIBSVM which is a library for SVM that is efficient for multi-class classification [13]. Overall (average) correct classification or accuracy might not always be applicable for multi-class classification because of possible dissimilar classification rates of different classes affecting the overall performance measure. Hence we measure the sensitivity $S$ of a given class from the confusion matrix $N$ following the scheme proposed in [14]. The number of patterns associated with class $i$ is given by $f_{i}$. The sensitivity $\left(S_{i}\right)$ of class $i$, estimates the number of patterns correctly identified to be in class $i$ with respect to the total number of patterns in class $i$, where $i=1 \ldots \mathrm{c}$ and c being the total number of classes. $N_{i j}$ represents the number of times that the patterns are identified to be in class $j$ when they really belong to class $i$. The necessary equations are:

$$
f_{i}=\sum_{j=1}^{c} N_{i j} \quad \text { and } \quad S_{i}=\frac{N_{i i}}{f_{i}} \times 100
$$

Given the significant degree of inter-person and temporal variability for the same movement within a population, the classifier needs to be robust enough to identify the same type of movements in the presence of large scale variability. The fundamental assumption is that if a pool of data encompassing large variability of a particular type of movement from a population is used to train a classifier then there is a significant chance that the classifier could then successfully identify that particular type of movement for a single subject, since there is a good probability that the characteristic movement of that subject is already embedded within the training dataset.

To test this hypothesis, we perform a 'leave-one-subjectout' validation methodology, where the process is repeated iteratively for all 18 subjects, each taking turn as the validation test set. Since each subject carries out one type of movement 20 times, for each sensor signal we have a data set consisting of 1440 samples $(18$ subjects $\times 20$ trials $\times 4$ movements). We keep one subject's data of 80 samples (1 subject $\times 20$ trials $\times 4$ movements) as the testing set and the remaining 1360 samples as the training set in each iteration to evaluate each of the three generalized classifiers for each of the 6 individual sensor signals, 2 modulus signals and 3 fused data signals. The three classifiers are assessed by comparing their largest sensitivities when successfully classifying all movements simultaneously, and the minimum number of features required to achieve these sensitivities.

\section{RESUlTS AND ANALYSIS}

The sensitivity for each arm movement recognised using the individual sensor data, their moduli and the fused data for each of the learning algorithms LDA, QDA and SVM are presented in Fig. 2 and summarised in Tables II, III and IV respectively. The sensitivity for each movement using the individual sensor signals for both the accelerometer and the gyroscope is better than that for the fused and the modulus signals. The difference in the recognitions rates between modulus and individual signals is partly due to the fact that bipolar information present in the raw data is retained with individual sensor signals, but lost with modulus signals. Hence, using the individual sensor signals provides the classifier an opportunity to select from a larger number of features and hence the recognition rate for the movements is reflected in the higher accuracies achieved. For the fused signals the sensitivity is generally lower when compared to results obtained from individual sensors, but better than that of the modulus signals. The sensitivity falls within $60-81 \%$ for the four movements for the fused signals with LDA and lies within 54-86\% with QDA and 50-75\% with SVM.

Considering LDA with individual sensor signals, the gyroscope recognises the four movements with sensitivities in the range of $83-96 \%$ while the accelerometer also has a similar detection rate with sensitivities in the range of 8491\% across all movements. However, the gyroscope uses only 12 features as compared to the 18 used by the individual sensor signals of the accelerometer out of a total of $30(3 \times 10$ features) and hence is the obvious choice with regard to a lower complexity solution. We can achieve a higher sensitivity (91\%) for Movement B using the accelerometer but that involves a cost of computing 6 extra features.

TABLE II. SUMMARY OF SENSITIVITIES FOR EACH MOVEMENT AND SIGNAL SOURCE FOR LDA CLASSIFIER.

\begin{tabular}{|l|c|c|c|c|c|}
\hline Signal & A (\%) & B (\%) & C (\%) & D (\%) & Features \\
\hline Acc_mod & 58 & 58 & 51 & 73 & 9 \\
\hline Acc_xyz & 85 & 91 & 84 & 90 & 18 \\
\hline Gyro_mod & 82 & 78 & 39 & 80 & 7 \\
\hline Gyro_xyz & 96 & 83 & 83 & 88 & 12 \\
\hline Fused & 81 & 74 & 60 & 75 & 13 \\
\hline
\end{tabular}

TABLE III. SUMMARY OF SENSITIVITIES FOR EACH MOVEMENT AND SIGNAL SOURCE FOR QDA CLASSIFIER.

\begin{tabular}{|l|c|c|c|c|c|}
\hline Signal & A (\%) & B (\%) & C (\%) & D (\%) & Features \\
\hline Acc_mod & 49 & 61 & 54 & 72 & 4 \\
\hline Acc_xyz & 89 & 92 & 78 & 91 & 15 \\
\hline Gyro_mod & 82 & 71 & 36 & 85 & 7 \\
\hline Gyro_xyz & 94 & 91 & 95 & 89 & 12 \\
\hline Fused & 86 & 72 & 54 & 74 & 11 \\
\hline
\end{tabular}


TABLE IV. SUMMARY OF SENSITIVITIES FOR EACH MOVEMENT AND SIGNAL SOURCE FOR SVM CLASSIFIER.

\begin{tabular}{|l|c|c|c|c|c|}
\hline Signal & A (\%) & B (\%) & C (\%) & D (\%) & Features \\
\hline Acc_mod & 42 & 53 & 55 & 70 & 5 \\
\hline Acc_xyz & 89 & 87 & 82 & 90 & 8 \\
\hline Gyro_mod & 90 & 74 & 35 & 80 & 5 \\
\hline Gyro_xyz & 97 & 85 & 90 & 89 & 11 \\
\hline Fused & 75 & 71 & 50 & 69 & 9 \\
\hline
\end{tabular}

A further comparison of the gyroscope results using individual sensor signals with QDA and SVM illustrates that the results for QDA and SVM are marginally higher than LDA, though the number of features required to successfully classify all four arm movements using individual sensor signals is similar for all three algorithms. Hence, in view of the trade-off between the recognition rate and the complexity involved, since LDA is considered to be computationally less complex [15], it appears to be the best choice classifier. A low complexity classifier is of particular relevance if software is to be translated in to a hardware format (custom chip) to produce a more energy efficient solution.
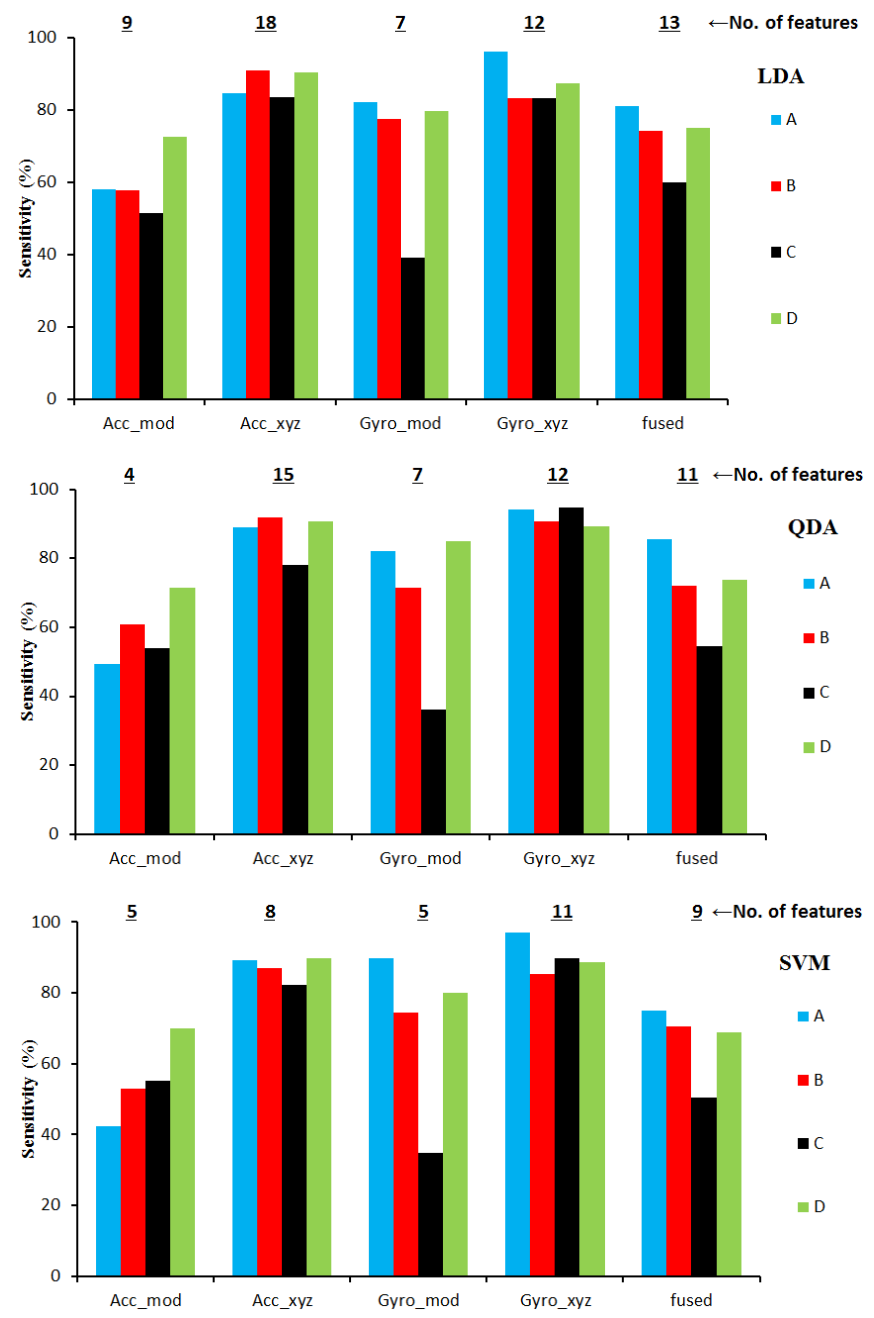

Fig. 2. Sensitivities for each arm movement using the accelerometer and gyroscope modulus signal (mod), individual sensor signals (xyz) and fused signals applied to the LDA, QDA and SVM classifiers. The number of features required for each signal group is shown at the top of each group.

\section{CONCLUSIONS}

In this paper we describe a systematic exploration on the appropriate selection of sensor type and classification strategy for detecting four fundamental types of human upper limb movement that are used in daily life activities, with particular attention to developing a robust training model accounting for significant inter-subject variability and evaluated with a 'leave-one-subject-out' validation process. We show that a tri-axial rate gyroscope placed on the forearm close to the wrist can independently classify all four movements with sensitivities in the range $83-96 \%$ when a small set of features (12) is extracted from the data from individual sensor axes in conjunction with LDA as the classification algorithm. The resulting 'best' activity classification system determined by this methodology is being implemented in our StrokeBack project to detect, classify and enumerate the various occurrences of prescribed exercises (i.e. arm movements) during normal daily activities, which over time can provide an indication of rehabilitation progress.

\section{REFERENCES}

[1] J. Parkka et al., "Activity classification using realistic data from wearable sensors," IEEE Trans. Info. Technol. Biomed., vol.10, no.1, pp.119-128, Jan. 2006.

[2] B. Najafi et al., "Ambulatory system for human motion analysis using a kinematic sensor: Monitoring of daily physical activity in the elderly," IEEE Trans. Biomed. Eng., vol. 50, no. 6, pp. 711-723, Jun. 2003.

[3] K. Maharatna et al., "Towards the development of next-generation remote healthcare system: Some practical considerations," in Proc. IEEE Int. Symp. Circuits and Systems (ISCAS), Seoul, pp. 1-4, May. 2012.

[4] F. E. Martınez-Pérez et al., "Activity inference for ambient intelligence through handling artifacts in a healthcare environment," Sensors, vol. 12, no. 1, pp. 1072-1099, Jan. 2012.

[5] C. Zhu and W. Sheng, "Motion- and location-based online human daily activity recognition," Pervasive and Mobile Computing, vol. 7, no. 2, pp. 256-269, Apr. 2011.

[6] A. Burns et al., "Shimmer - A wireless sensor platform for noninvasive biomedical research," IEEE Sensors Journal, vol. 10, no. 9, pp.1527-1534, Sep. 2010.

[7] C. Kendell and E. D. Lemaire, "Effect of mobility devices on orientation sensors that contain magnetometers," J. Rehab. Res. Dev., vol. 46, no.7 pp. 957-962, 2009.

[8] O. Banos et al., "Daily living activity recognition based on statistical feature quality group selection," Exp. Syst. Appl., vol. 39, no. 9, pp. 8013-8021, July 2012.

[9] S. Chernbumroong et al., "Elderly activities recognition and classification for applications in assisted living," Exp. Syst. Appl., vol. 40, no. 5, pp. 1662-1674, Apr. 2013.

[10] I. Kim et al., "ADL classification using triaxial accelerometers and RFID," in Proc. $1^{\text {st }}$ Int. Conf. Ubiq. Comp. Conver. Technol., 2007.

[11] S. Patel et al., "A novel approach to monitor rehabilitation outcomes in stroke survivors using wearable technology," Proceedings of the IEEE, vol. 98, no. 3, pp. 450-461, Mar. 2010.

[12] S. Theodoridis and K. Koutroumbas, "Pattern Recognition", $4^{\text {th }}$ ed., Elsevier, pp. 79-135, 2008

[13] LIBSVM - A Library for Support Vector Machines. 2013. [Online] Available at: http://www.csie.ntu.edu.tw/ cjlin/libsvm/

[14] J. C. F. Caballero et al., "Sensitivity versus accuracy in multiclass problems using memetic pareto evolutionary neural networks," IEEE Trans. Neural Networks, vol. 21, no. 5, pp. 750-770, May 2010.

[15] T. Chen et al., "On the trade-off of accuracy and computational complexity for classifying normal and abnormal ECG in remote CVD monitoring systems," in Proc. IEEE Workshop Signal Processing Systems (SIPS), Quebec, pp. 37-42, Oct. 2012. 